\title{
Low-intensity training provokes adaptive extracellular matrix turnover of a muscular dystrophy model
}

\author{
Thaís P. Gaiad 1,* , Murilo X. Oliveira', Adalfredo R. Lobo-Jr.', Lívia R. Libório', Priscilla A.F. Pinto', Danielle C. Fernandes', \\ Ana Paula Santos', Carlos Eduardo Ambrósio ${ }^{3}$, Alex Sander D. Machado ${ }^{4}$ \\ 'Department of Physical Therapy, Universidade Federal dos Vales do Jequitinhonha e Mucuri (UFVJM), Diamantina, Brazil \\ ${ }^{2}$ Institute of Agriculture Scinces, Universidade Federal dos Vales do Jequitinhonha e Mucuri (UFVJM), Unaí, Brazil \\ ${ }^{3}$ Department of Veterinary Medicine, University of São Paulo (USP), Pirassununga, Brazil \\ ${ }^{4}$ Faculty of Medicine FAMED, Universidade Federal dos Vales do Jequitinhonha e Mucuri (UFVJM), Diamantina, Brazil
}

Recommendations of therapeutic exercise in Duchenne muscular dystrophy are still controversial. The hypothesis that a low-intensity training (LIT) protocol leads to muscle adaptations on mdx mice model was tested. Dystrophic male mice with 8 weeks old were separated in exercised $(m d x E, n=8)$ and sedentary $(m d x C, n=8)$ groups. Wild-type mice were used as control (WT, $n=8)$ group. Exercised group underwent a LIT protocol ( $9 \mathrm{~m} / \mathrm{min}, 30 \mathrm{~min}, 3$ days/wk, 60 days) on a horizontal treadmill. At day 60 all animals were analyzed regarding parameters of markers of muscle lesion and extracellular matrix turnover of muscle tissue by collagens fibers on tibial anterior muscle. Histomorphometry attested that centrally located nuclei fibers and the coefficient of variance of minimal Feret's diameter was similar in $\mathrm{mdxE}$ and $\mathrm{mdxC}$ groups $(P=1.000)$ and both groups presented higher mean values than WT group $(P<0.001)$.
Fraction area of collagen fibers of $\mathrm{mdxE}$ group was lower than $\mathrm{mdxC}$ group $(P=0,027)$ and similar to WT group $(P=0,751)$. Intramuscular area of $\mathrm{Col} 3$ of the $\mathrm{mdxE}$ group was higher than $\mathrm{mdxC}$ and WT groups $(P<0.001)$. Intramuscular area of Col1 on the mdxE group was similar to the $\mathrm{mdxC}$ group $(P=1.000)$ and both groups were higher than WT group $(P<0.001)$. LIT protocol had not influenced muscle injuries resulting from the dystrophin-deficiency membrane fragility. Although, LIT had provoked adaptations on extracellular matrix bringing higher elastic feature to dystrophic muscle tissue.

Keywords: Collagen type I, Collagen type III, Exercise therapy, Extracellular matrix, Mice mdx, Skeletal muscle

\section{INTRODUCTION}

Animal models for Duchenne muscular dystrophy (DMD) have brought significant insights in the field of therapeutic exercise prescription and preclinical standards measurements (Aurora et al., 2014; Frinchi et al., 2014; Gaiad et al., 2012; Smythe and White, 2011). The mdx mouse is the most common animal model with genetic and biochemistry homology and its progression phenotype is considered moderate (Vainzof et al., 2008). Although the standard mdx mouse has some deficiencies, including its almost normal lifespan and mild dysfunction, it is widely used as a model of DMD, partly due to its convenience, availability and genetic comparability with the human disease (Manning and O'Mal- ley, 2015).

Healthy myofibres have peripheral nuclei, intact sarcolemma and nonfragmented sarcoplasm. In turn, dystrophic muscle is characterized by the presence of infiltrating inflammatory cells, hypercontracted myofibres, degenerating myofibres with fragmented sarcoplasm, and centrally located nuclei (CLN) (regenerated myofibres) (Pertl et al., 2013).

A progressive replacement of muscle by fibrous connective tissue (mild fibrosis) is reported in limb muscles from 10-13 weeks, with extensive fibrous connective tissue (fibrosis) and some calcification from 16 to 20 months of age (Grounds et al., 2008). It is known that fibrosis occurs in skeletal muscle secondary to injury and disease and is generally defined as an increase area of extracel-
${ }^{*}$ Corresponding author: Thaís P. Gaiad (1) https://orcid.org/0000-0002-4688-330X Physical Therapy Department, Universidade Federal dos Vales do Jequitinhonha e Mucuri (UFVJM), Rod MGT 367 - km 583, n 5000, CEP: 39100-000 -

Diamantina, MG, Brazil

Tel: +55-38-3532-1239, E-mail: thaispgm@gmail.com

Received: August 28, 2017 / Accepted: December 5, 2017
This is an Open Access article distributed under the terms of the Creative Commons Attribution Non-Commercial License (http://creativecommons.org/licenses/by-nc/4.0/) which permits unrestricted non-commercial use, distribution, and reproduction in any medium, provided the original work is properly cited. 
lular matrix (ECM) muscle cross-sections (Gillies et al., 2017). In vivo techniques have underlined that connective tissue of skeletal muscle and tendon is a lively structure with a dynamic protein turnover and that it possesses the capacity to adapt greatly to changes in the external environment such as mechanical loading or inactivity and disuse (Kjaer et al., 2006). Determining alterations in ECM organization due to disease or injury is also clinically relevant since these alterations may have functional consequences (Gillies et al., 2017; Kjaer et al., 2006).

There is no cure available and the current management of the DMD is based on prevention and management of complications (Haas et al., 2015). Recently, Bushby et al. (2014) published the results of the effects of Ataluren treatment of patients with nonsense mutation dystrophinopathy. This finding and the review conduct by a European group (Haas et al., 2015) has shown that it is a promise treatment for this genetic disorder.

Patients are used forwarded to rehabilitation programs in which continuous physiotherapy plays a vital role. Although, motor physical therapy has controversial recommendations (Bushby et al., 2010; Carter et al., 2002) and there is no consensus about the best type or intensity of therapeutic exercise (Bushby et al., 2010; Cup et al., 2007). According to a systematic review about the efficacy of muscle exercise in patients with muscular dystrophy conduct by Gianola et al. (2013), there is simply no evidence about the type, frequency, and intensity of exercise. Moreover, detrimental effects of exercise on muscular dystrophy also remain a possibility.

Low-intensity training (LIT) on treadmill at $9 \mathrm{~m} / \mathrm{min}$ have been previous assessed on murine model of DMD analyzing its effects on physiological adaptation showing a reduction on markers of oxidative stress, increasing mitochondrial capacity and on the inflammatory status of mdx mice (Hyzewicz et al., 2015; Hyzewicz et al., 2017; Kaczor et al., 2007).

The effects of a LIT protocol on morphological markers of skeletal muscle degeneration, such as CLN counting, coefficient of variance $(\mathrm{CV})$ of minimal Feret's diameter; and compounds of the ECM as fraction area of total collagen fibers and local rearrangement of types I and III collagen to attest functional features of the dystrophic skeletal muscle adaptation were not analyzed yet. CLN is a parameter widely used in $\mathrm{mdx}$ model studies to demonstrate the efficacy of preclinical studies of DMD (Briguet et al., 2004; Dubach-Powell, 2014). Muscular fibrosis is also a result of an aberrant process of muscle repair in dystrophic muscle (Gosselin et al., 2007; Mann et al., 2011) and types I and III collagen are two common types of collagen fibers that have been investigated to verify the influence of different therapies on skeletal dystrophic muscle (Gaiad et al., 2014; Huebner et al., 2008; Turgeman et al., 2008). The location of these types of collagen fibers in dystrophic muscle through immunohistochemistry analysis and the quantification of their proportion in the skeletal muscle can bring some knowledge about the dystrophic muscle repair submitted to LIT protocol.

Aiming to find a better way to maintain the muscular activity without muscle damage, the hypothesis that 60 days of horizontal treadmill LIT protocol provoke beneficial muscle repair on $\mathrm{mdx}$ mice was tested.

\section{MATERIALS AND METHODS}

\section{Experimental design}

Sixteen male mdx mice (C57BL/10ScSn-Dmdmdx/J) and eight wild-type mice (C57BL/10) acquired from FioCruz Institute (Rio de Janeiro, Brazil), were used in this study. This research was approved by the Ethics Committee on Animal Use of the University Federal dos Vales do Jequitinhonha e Mucuri, protocol number $017 / 2011$. The rodents were maintained in cages on a $12 \mathrm{hr}$ day/12 hr dark cycle at $22^{\circ} \mathrm{C}$ and supplied with food and water ad libitum. The mdx mice were randomized into two groups: Exercised group ( $m d x E, n=8)$ and sedentary group $(m d x C, n=8)$. A control group was composed by wild-type mice and denominated WT $(\mathrm{n}=8)$. The animals started the exercise protocol at 8 weeks old, once at this age mdx mice have already suffered cycles of degeneration-regeneration and due to the similarities of fibrosis and muscular deterioration between humans and $\mathrm{mdx}$ mice age-matched (Grounds et al., 2008).

\section{LIT protocol}

The exercise protocol started at the day after the first assessment (zero) of body weight. The animals of $\mathrm{mdxE}$ group underwent a LIT protocol on a horizontal treadmill (EP 131; Insight, Ribeirão Preto, Brazil) $30 \mathrm{~min} /$ day, 3 times a week for 60 days at a velocity of $9 \mathrm{~m} / \mathrm{min}$. The protocol speed was controlled at $9 \mathrm{~m} / \mathrm{min}$ so that the therapeutic training was considered of low intensity (Grange, 2014). Before the experimental period, the animals underwent a period of adaptation in order to familiarize with the apparatus and speed, which was gradually increased. Aiming to offer the same environmental stimuli to experimental mice, the animals of $\mathrm{mdxC}$ group were placed at the off treadmill $(0 \mathrm{~m} / \mathrm{min})$ with the same duration and frequency of the animals of $\mathrm{mdxE}$ group.

Body weight (g) of all animals was assessed over the time of the exercise protocol $(0,15,30,45$, and 60 days). On the 60th day of 
protocol, samples of tibialis anterior (TA) muscle were harvested for analysis. Morphological, histochemical and immunohistochemistry analysis were all conducted in this same muscle group.

\section{Histological and histochemical analysis}

The muscle samples were fixed in paraformaldehyde solution at $4 \%$, treated with increasing ethanol concentrations ( $70 \%$ to $100 \%$ ) to dehydrate and with xylene to clear. The samples were then embedded in paraffin and sections of $5 \mu \mathrm{m}$ in thickness were obtained. The cross-sections were oven-dried $\left(60^{\circ} \mathrm{C}\right)$ at a horizontal position. After deparaffinization protocol, the sections were stained using hematoxylin and eosin (H\&E) to identify histopathological features. Histochemical reaction using Picrossirius red, a combination of Sirius red F3BA (Colour Index 35780, Sigma-Aldrich, St. Louis, MO, USA) dissolved in a saturated picric acid solution, was used in order to distinguish collagen from the skeletal muscle fibers. Photomicrographs were made under an optical microscope (LABOMED LxPol, Labo America, Fremont, Canada) equipped with an Axio CAM HRc camera and Software Capture Pro 2.9.0.1.

\section{Immunohistochemistry analysis}

Primary polyclonal antibodies against collagen (anti-mouse) types I (ab34710, Abcam, Cambridge, MA, USA) and III (ab7778, Abcam) (1:100 and 1:250 dilution) were applied on the muscle sections, separately. Sections were immersed in citric acid solution at $0.01 \mathrm{M}, \mathrm{pH} 6.0$ and submitted to $95^{\circ} \mathrm{C}$ for $30 \mathrm{~min}$ to for antigenic recovery. After, the blockade of endogenous peroxidase with hydrogen peroxide at $3 \%$ for 30 min were performed. Primary antibodies were applied and sections were incubated for $20 \mathrm{hr}$ in a damp chamber at $4^{\circ} \mathrm{C}$. After three more rinses in PBS, secondary antibody (NIC-414341F, N-Histofine Simple Stain MAX PO, Carlsbad, CA, USA) was applied and incubated for $20 \mathrm{~min}$ at room temperature $\left(24^{\circ} \mathrm{C}\right)$. Immunohistochemistry (IHC) reaction was revealed with DAB (Chromogen/Substrate Bulk Pack, ScyTek Laboratories, West Logan, UT, USA) for 5 min. Photomicrographs were taken using an optical microscope (LABOMED LxPol, Labo America) equipped with an Axio CAM HRc camera and Software Capture Pro 2.9.0.1.

\section{Qualitative sample analysis}

Qualitative assessments of histological, histochemical and IHC analysis of TA muscle samples were made by observing three sections from each one of the animals $(\mathrm{n}=8)$ per group.

\section{CLN quantification}

The total number of CLN were determined from HE stained sections from the muscle samples. Thirty nonoverlapping $\times 100$ images from each group were taken from TA muscle (about ten hundred muscle fibers/group). The percentage of the total number of CLN was obtained by normalizing number of CLN counted to the total number of fibers per image using ImageJ software (National Institute Health, Bethesda, MD, USA).

\section{Minimal Feret's diameter}

The minimal Feret's diameter is the minimum distance of parallel tangents at opposing borders of the muscle fiber (Briguet et al., 2004). The CV of the minimal Feret's diameter was determined from $\mathrm{H} \& \mathrm{E}$ stained sections from $\times 400$ images from the muscle samples. CV of a minimum of 100 muscle fibers per animal was analyzed (De Brito et al., 2006) using the ImageJ software. Only fibers with integrity of sarcolemma were counted. Results are present as mean values of the individual coefficient of variation, according to previously published studies (Briguet et al., 2004; Dubach-Powell, 2014).

\section{Intramuscular collagen fiber quantification}

To accurately quantify total collagen-positive areas, slides reacted with picrossirius red from fifteen randomly selected fields $\times 100$ per animal ( 2,000 fibers/group) were quantified (Smith and Barton, 2014). Moreover, the fraction area of collagenous tissue determined from immunohistochemistry of types I and III collagen stained sections of thirthy nonoverlapping images $\times 400$ were also quantified, according to Ardite et al. (2012).

The images were converted to 8 bit (blue), the background was subtracted and the images were automatically scaled from 0 to 255. Before that, a threshold was applied to each image using Image J software. Data of each type of intramuscular collagen are shown in percentage of the total area $\left(57,247.67 \mu \mathrm{m}^{2}\right)$.

\section{Statistical analysis}

An analysis of variance (ANOVA) was performed for the data of body weight using a completely randomized design (CRD) in a 3 (groups) $\times 5$ (time of LIT protocol) factorial arrangement with eight repetitions (animals) in each group. Those variables were analyzed as repeated measures on time, since the measures taken on the same animal over the time are correlated. In turn, an ANOVA was also performed for the data of CLN and intramuscular area of collagen (Col1 and $\mathrm{Col} 3$ ) using a CRD, in which only effect of group was included in the model. When a significant in- 
teraction $(P \leq 0.05)$ between group (qualitative) and time of LIT protocol (quantitative) was detected, a slicing was performed to verify at what levels there were differences. The Student $t$-test was used to discriminate the least squares means of the groups. The data was analyzed using the MIXED procedures of the SAS ver. 9.2 (SAS Institute Inc., Cary, NC, USA).

\section{RESULTS}

The animals of the mdxE group completed the LIT protocol 60 days after LIT protocol start. Mean values of body weight $(\mathrm{g})$ were maintained $(P<0.05)$ over the time of LIT protocol within each dystrophic group, without significant difference (Fig. 1).

It is possible to observe in Fig. 2 that the TA muscle of the WT group presents thin tracts of endomysium and perimysium between muscle fibers and between muscle fascicles, respectively.

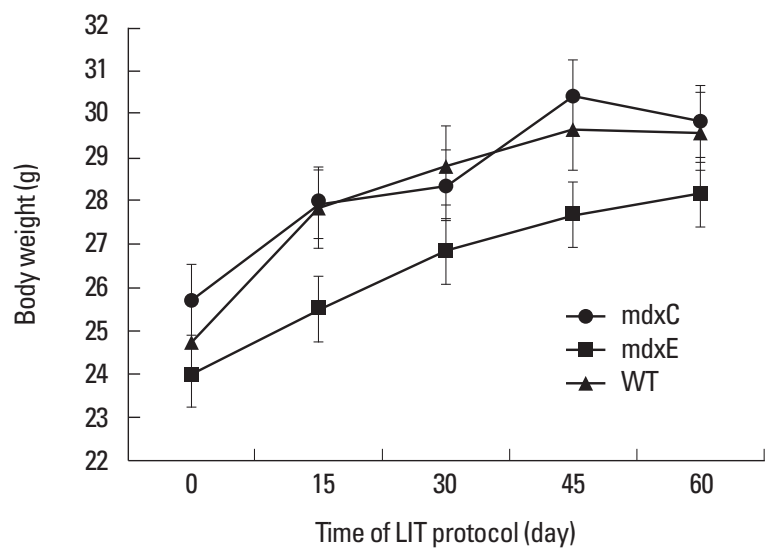

Fig. 1. Body weight over the time of low-intensity training (LIT) protocol. Mean values and standard error of body weight $(\mathrm{g})$ of $\mathrm{mdxC}, \mathrm{mdxE}$, and $\mathrm{WT}$ over the time of LIT protocol. Mean values of body weight $(\mathrm{g})$ were maintained $(P<0.05)$ over the time of LIT protocol within each dystrophic group, without significant difference. $\mathrm{mdxC}$, mdx mice sedentary group; $\mathrm{mdxE}$, $\mathrm{mdx}$ mice exercised group; WT, wild-type mice.
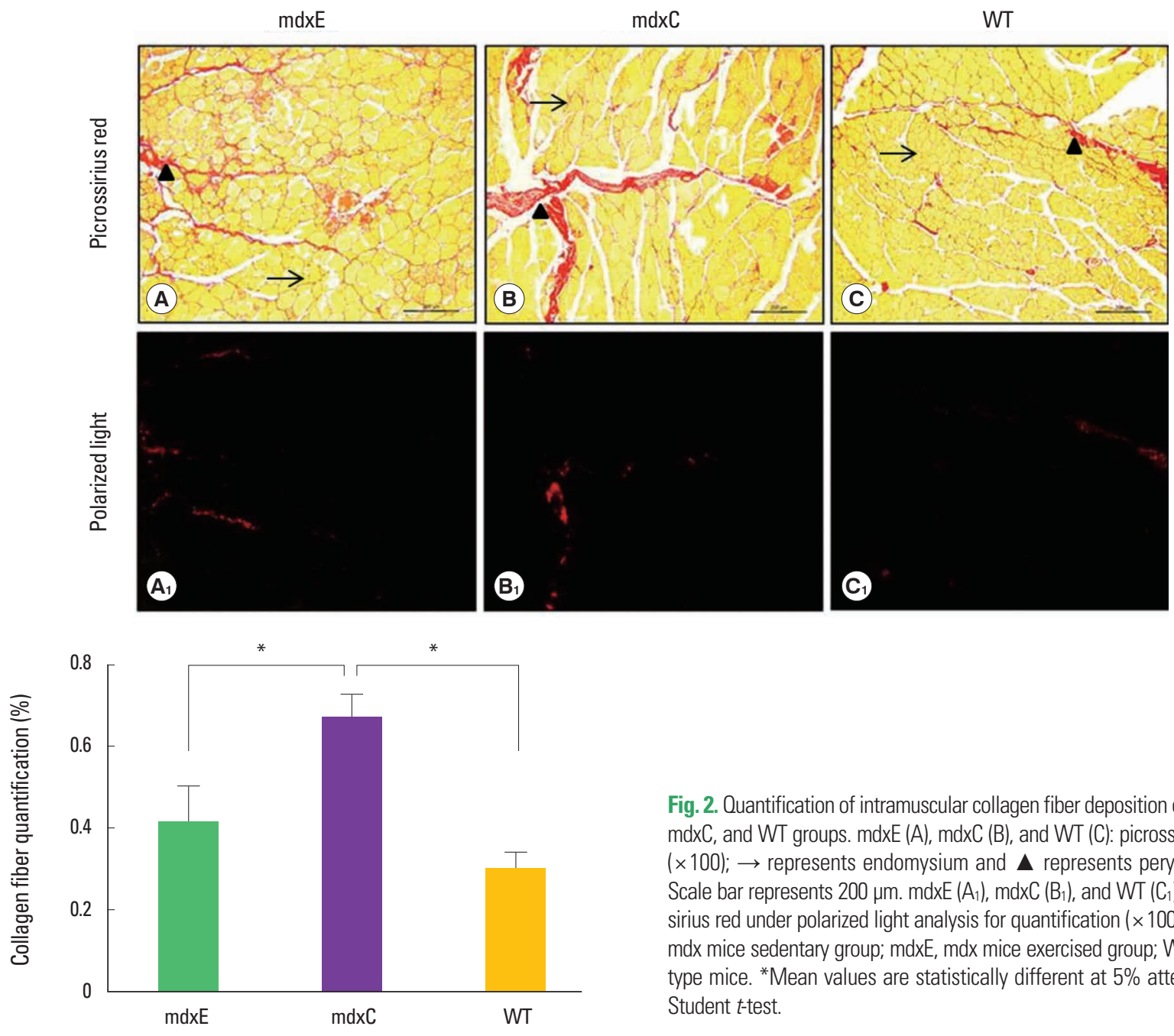

Fig. 2. Quantification of intramuscular collagen fiber deposition of mdxE, $m d x C$, and $W T$ groups. $m d x E(A)$, mdxC (B), and WT (C): picrossirius red $(\times 100) ; \rightarrow$ represents endomysium and $\boldsymbol{\Delta}$ represents perymisium. Scale bar represents $200 \mu \mathrm{m}$. $\operatorname{mdxE}\left(A_{1}\right), \operatorname{mdxC}\left(B_{1}\right)$, and $W T\left(C_{1}\right)$ : picrossirius red under polarized light analysis for quantification $(\times 100)$. $m d x C$, $\mathrm{mdx}$ mice sedentary group; $\mathrm{mdxE}$, mdx mice exercised group; WT, wildtype mice. *Mean values are statistically different at $5 \%$ attested by Student $t$-test. 
Quantitative analysis shows that the percentage of intramuscular collagen fibers was significate different between groups $(P=0.003)$. Mean values of $\mathrm{mdxE}$ group $(0.41 \% \pm 0.07 \%)$ was lower than $\operatorname{mdxC}$ group $(0.66 \% \pm 0.06 \%)(P=0.042)$ and similar to WT group $(0.30 \% \pm 0.04 \%)(P=0.761)$. Mean values of $\mathrm{mdxC}$ group was also significate different of WT group $(P=0.003)$. The higher amount of this fraction area of collagen fibers of $\mathrm{mdxC}$ group can be observed mainly on perimysium structures (Fig. 2).

Analysis of TA muscle of WT group shows peripheral nuclei and homogeneity of muscle fibers (Fig. 3). Muscle with CLN and heterogeneity of muscle fibers were found in the dystrophic groups at day 60 of LIT protocol, however dystrophic muscle of $\mathrm{mdxC}$ group presented also areas with inflammatory infiltrate and necrosis. No difference was observed in the percentage of CLN between the $\mathrm{mdxC}(70.0 \% \pm 2.15 \%)$ and the $\mathrm{mdxE}(67.6 \% \pm 1.63 \%)$ groups $(P>0.05)$, but both groups had higher CLN than the WT group $(3.1 \% \pm 1.76 \%)(P<0.05)$. The mean values of minimal Feret's diameter $\mathrm{CV}$ was similar on $\mathrm{mdxC}$ group $(0.316 \pm 0.009)$ and
mdxE group $(0.314 \pm 0.017)$ and both different and higher than WT group (0.244 \pm 0.018$)$ (Fig. 3).

$\mathrm{Col} 3$ was located only at perimysium structures of WT group staining thin tracts. In $\mathrm{mdxE}$ and $\mathrm{mdxC}$ groups, $\mathrm{Col} 3$ was observed at perimysium and also at endomysium structures (Fig. 4). Intramuscular area of $\mathrm{Col} 3$ of the $\mathrm{mdxE}$ group $(9.7 \% \pm 0.58 \%)$ was higher than the $\mathrm{mdxC}$ group $(5.3 \% \pm 0.58 \%)$ and the WT group $(4.7 \% \pm 0.58 \%)(P<0.05)$, with no difference between the $\mathrm{mdxC}$ group and the WT group $(P>0.05)$ (Fig. 4). The higher amount of $\mathrm{Col} 3$ intramuscular area of mdxE group can be observed mainly on endomysium structures (Fig. 4).

Immunohistochemical analysis demonstrated that Coll was quietly observed at endomysium and perimysium structures on WT group as thin tracts. Intramuscular area of Col1 was similar to $m d x E$ group $(15.5 \% \pm 0.9 \%)$ and $m d x C$ group $(16.5 \% \pm 0.9 \%)$ $(P>0.05)$ and both groups were higher than WT group $(9.9 \% \pm$ $0.9 \%)(P<0.05)$ (Fig. 5).
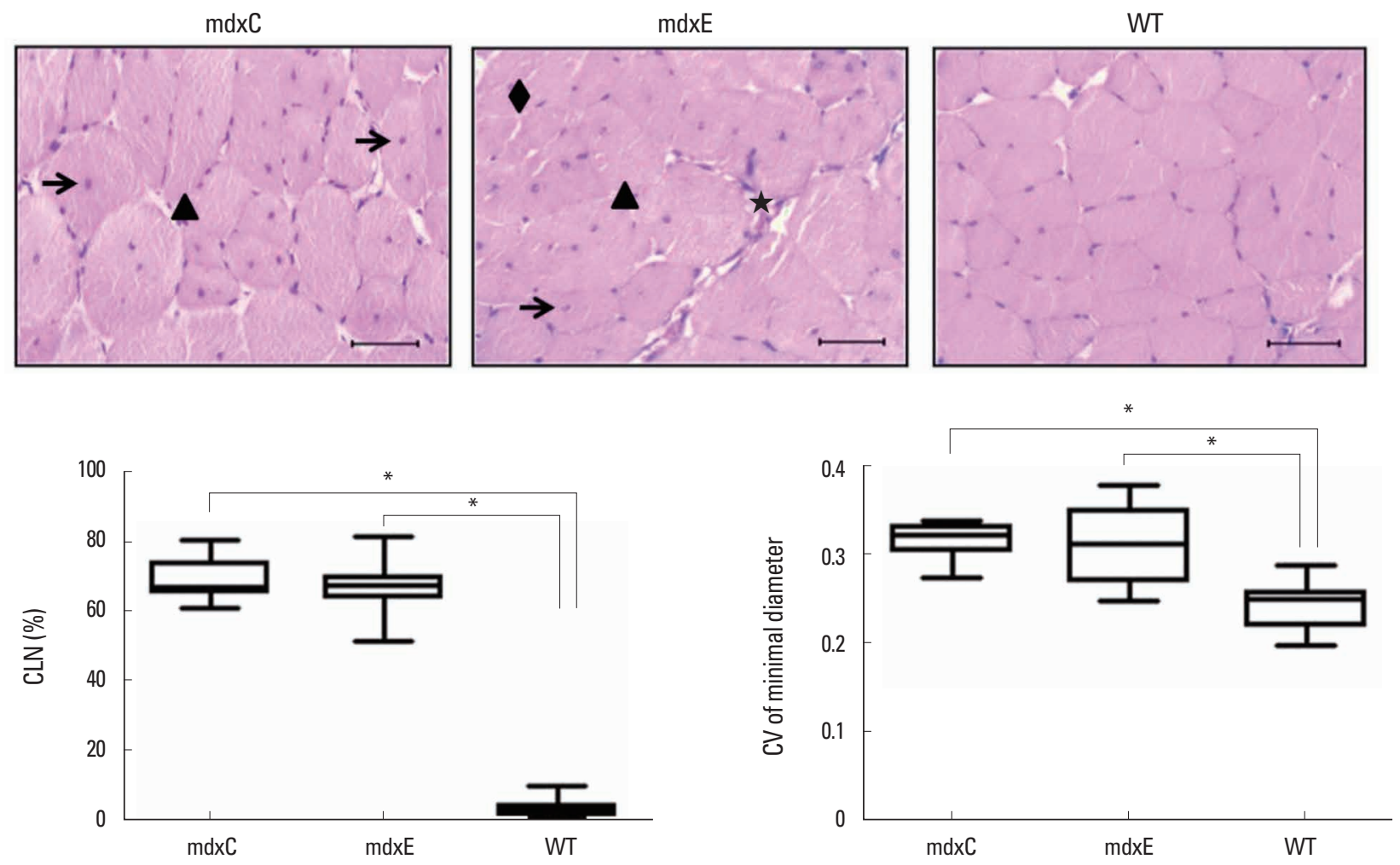

Fig. 3. Upper panel: Morphological analysis of tibialis anterior muscles of $m d x C, m d x E$, and WT groups at 60 days of low-intensity training protocol (H\&E, $\times 400)$. represents heterogeneity of muscle fibers, $\rightarrow$ represents centrally located nuclei, $\star$ represents inflammatory infiltrate, and $\downarrow$ represents necrosis. Scale bar represents $40 \mu \mathrm{m}$. Lower panel: Percentage of muscle fibers with centrally located nuclei (CLN) and coefficient of variance (CV) of Minimal Feret's Diameter. Mean values of $m d x C$ and $m d x E$ are statistically different from mean values of WT groups in both graphics. mdxC, mdx mice sedentary group; mdxE, mdx mice exercised group; WT, wild-type mice. * Mean values are statistically different at $5 \%$ attested by Student $t$-test. 

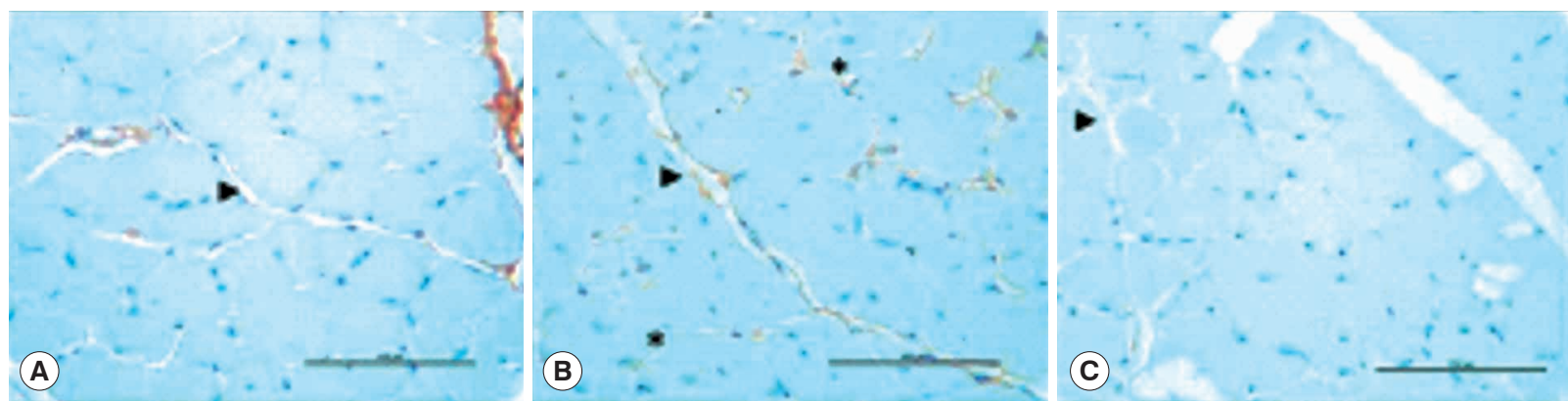

Fig. 4. Upper panel: Photomicrographs of immunohistochemistry analysis of collagen III and quantification of intramuscular collagen III area in tibialis anterior muscles $(\times 400)$. Positive immunostaining for collagen type III (1:250) of $\operatorname{mdxC}(A), \operatorname{mdxE}(B)$, and WT (C) groups. Scale bar represents $100 \mu \mathrm{m}$. Col3 stained at perimysium ( $)$ and endomysium tracts $\left({ }^{*}\right)$. Lower panel: Graphic of the effect of 60 days of low-intensity training on \% of intramuscular Col3 area between $m d x C$, $m d x E$, and WT groups. $m d x C$, mdx mice sedentary group; mdxE, mdx mice exercised group; WT, wild-type mice. ${ }^{\text {a,bl}}$ Mean values followed by different letters show statistical difference between them at $5 \%$ attested by Student $t$-test (standard error of the mean, 0.58 ).
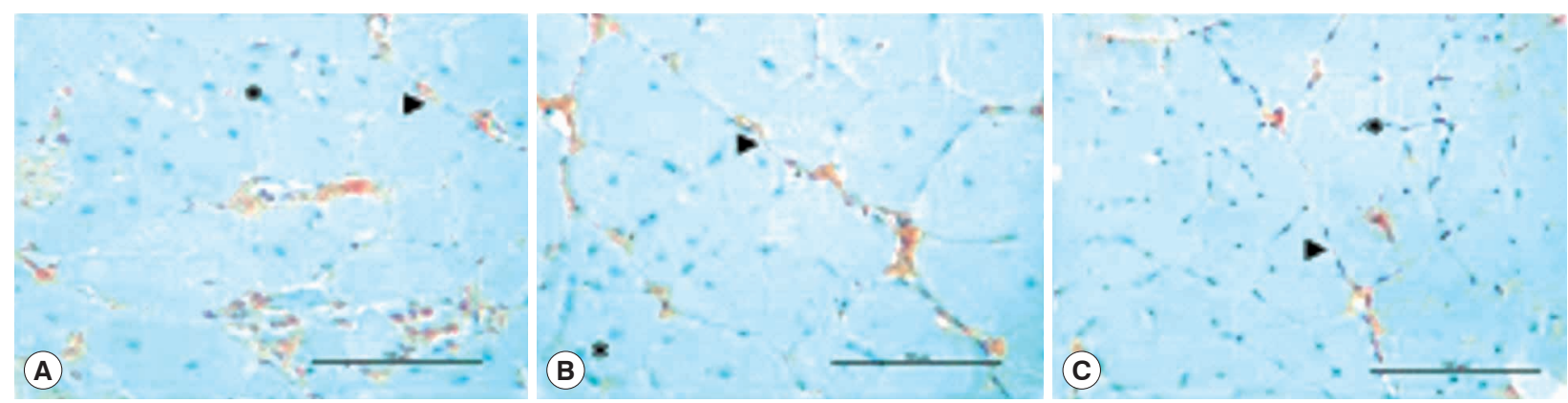

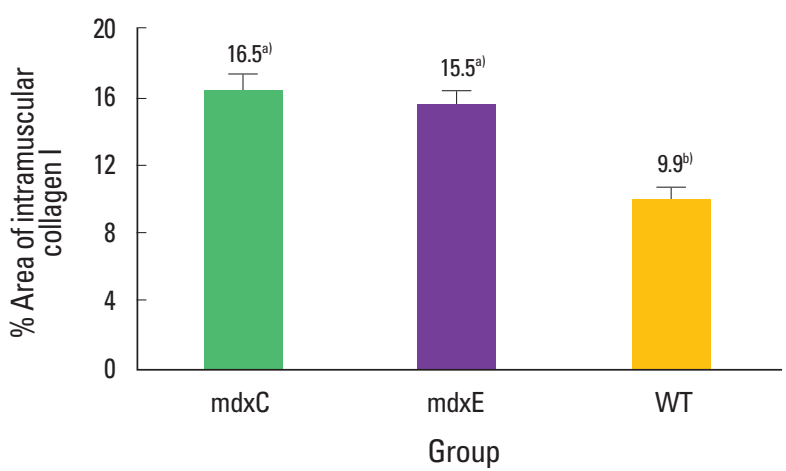

\section{DISCUSSION}

The LIT protocol here employed has shown that exercise can act in a beneficial way on dystrophic skeletal muscle repair to ex-
Fig. 5. Upper panel: Photomicrographs of immunohistochemistry analysis of collagen I and quantification of intramuscular collagen I area in tibialis anterior muscles $(\times 400)$. Positive immunostaining for collagen type I (1:100) of mdxC (A), mdxE (B), and WT (C) groups. Scale bar represents $100 \mu \mathrm{m}$. Col1 stained at perymisium ( $)$ and endomysium tracts $\left(^{*}\right)$. Lower panel: Graphic of the effect of 60 days of low-intensity training on $\%$ of intramuscular Col1 area between $m d x C, m d x E$, and WT groups. $m d x C$, mdx mice sedentary group; $m d x E$, $m d x$ mice exercised group; WT, wild-type mice. ${ }^{\text {a,b }}$ Mean values followed by different letters show statistical difference between them at $5 \%$ attested by Student $t$-test (standard error of the mean, 0.90).

ercise. The type III collagen fiber location and amount associated with the decreased amount of intramuscular collagen fibers and maintained muscle regeneration tells that LIT delayed the aberrant repair at the studied age. 
It is well established among physiotherapists that eccentric contractions are not recommended to human DMD (Gianola et al., 2013) and high-resistance training is far controversial and questionable (Markert et al., 2011). On the other hand, inactivity is not recommended to humans with DMD because it aggravates muscle weakness and susceptibility to contraction-induced damage (Ferry et al., 2015). Recent studies on the mdx animal model of DMD (Call et al., 2010; Hyzewicz et al., 2015; Hyzewicz et al., 2017) have shown that some sort of activities can be beneficial to dystrophic skeletal muscle and they are necessary. Although, the type of exercise, frequency and intensity of the protocol must yet be defined aiming to really provoke positive changes in dystrophic muscle (Gianola et al., 2013).

According to different studies, LIT protocol seems to be the intensity able to attenuate the phenotype of $\mathrm{mdx}$ mice. These studies have investigated the effects of LIT at the physiological or molecular level, but none have investigated ECM turnover. Moreover, authors have used different modalities of exercise training as treadmill (Kaczor et al., 2007), swimming (Hyzewicz et al., 2015; Hyzewicz et al., 2017), Rota Rod (Frinchi et al., 2014) and wheel running (Baltgalvis et al., 2012).

Age of the animals, muscle group chosen for analysis, frequency and duration of the LIT protocol vary among these studies, which make it difficult to understand what are the real benefits of this intensity of exercise training to $\mathrm{mdx}$ model.

Exercise has different impacts on different muscles (depending on which muscles are recruited for the specific exercises regimes). TA muscle is a hind limb muscle that has been widely used to investigate the effects of different exercise protocols on mdx mice, is highly susceptible to contraction-induced Injury (Dellorusso et al., 2001) and has great percentage of type II fibers (Grounds et al., 2008). TA muscle contains predominantly types IIB and IIDB fibers, while types IIAD, IID, IIA, I and IC/IIC fibers were less abundant (Augusto et al., 2004) Fast-twitch muscles are generally the most susceptible to muscular dystrophy (and ageing) in all species (Lynch et al., 2001). Therefore, this is a reliable muscle to be analyzed while studying the effects of an exercise protocol on skeletal muscle morphological-functional features.

Exercise parameters such as speed, duration and angle of the treadmill are strictly controlled at horizontal treadmill training (Grange, 2014). Once we intent to provoke beneficial adaptations to skeletal dystrophic muscle repair, a speed of $9 \mathrm{~m} / \mathrm{min}$ was used. Also, it was followed the standard procedures for pre-clinical testing in the mdx mouse model of DMD classified by Grounds et al. (2008) as B3: 'Long term studies' in young adult mice where exer- cise started at 8 weeks of age and sampling performed at 16 weeks.

The study conducted by Kaczor et al. (2007) also used the treadmill to the LIT protocol (type of exercise) and the same speed of 9 $\mathrm{m} / \mathrm{min}$. Different from our research they studied younger $\mathrm{mdx}$ mice of 28 days of life, 2 times a week. They observed that this LIT protocol induced physiological adaptation resulting in lower levels of oxidative stress markers. Nevertheless they have not analyzed the impact of their protocol directly on tissue repair, as morphological markers of muscle degeneration, intramuscular fibrosis deposition or ECM turnover

Among other molecular and physiological parameters, Capogrosso et al. (2017) have assessed the effects of a long protocol of exercise (at $12 \mathrm{~m} / \mathrm{min}$ ) on the histopathology of hind limb muscles of younger mdx mice with 4-5 weeks age. As observed in our results, they detected the typical histological features of dystrophic muscle in the exercised group, and no detectable difference was observed in the percentage of CLN myofibers between exercised and sedentary $\mathrm{mdx}$ groups. The level of transforming growth factor- $\beta 1$ and plasmatic levels of matrix metalloproteinase- 9 confirmed the persistence of muscle damage in $\mathrm{mdx}$ mice. Therefore, dystrophic muscles showed a partial degree of functional adaptation to chronic exercise, although not sufficient to overcome weakness or signs of damage.

With the same speed of the study of Capogrosso et al. (2017), Schill et al. (2016) investigated a 4 weeks of twice-weekly treadmill exercise on mdx mice of 4 weeks old, younger than ours. The exercise regimen consisted of $30 \mathrm{~min}$ on a horizontal treadmill running at a speed of $12 \mathrm{~m} / \mathrm{min}$. They found that exercised $\mathrm{mdx}$ mice exhibit increased oxidative stress, as well as deficits in exercise capacity, baseline oxygen consumption and increased myofibres fibrosis attested by collagen I content.

These last researches conducted by Capogrosso et al. (2017) and Schill et al. (2016) showed that the high intensity exercise caused an adaptation not ideal for the dystrophic muscle, confirming the goal of this intensity already established by The TREAT-NMD recommended protocol "Use of treadmill and wheel exercise for impact on mdx mice phenotype." So, few researches until now have analyzed treadmill protocols of low intensity that seems to be beneficial to mdx mice muscle.

Young mdx mice at the acute phase of dystrophinopathy have muscle weakness and their muscles appear more susceptible to fatigue in vivo when compared to control mice. This acute phase of necrosis peak, with TA muscle being affected in 30\%-60\%, occurs at 25 to 26 days of life and then decreases significantly to stabilize at 8 weeks of age to a relatively low-level of active damage 
( 4\%-6\%) (Grounds et al., 2008; Willmann et al., 2012). The exercised mdx mice started the LIT protocol at 8 weeks of age, a phase of low-level of active damage that allows considerations regarding the maintenance of the features of dystrophinopathy on muscle morphology and function, once mdx mice present established dystrophy features at this age.

Exercised (67.6 \pm 1.63$)$ and sedentary $\mathrm{mdx}(70 \pm 2.15)$ groups at 60 days of LIT ( 16 weeks old, $\approx 4$ months), presented mean values of TA centrally located fibers similar to the ones presented by the study of Pertl et al. (2013). According to these authors, CLN fibers and the $\mathrm{CV}$ of the minimal Feret's diameter describe the process of degeneration and regeneration in $\mathrm{mdx}$ mice and the high degree of variability in the myofibre size because of a higher number of smaller fibers and the occurrence of hypertrophic fibers (Briguet et al., 2004). It suggest that the muscle of the mdx animals went through a regeneration process recently and confirmed that LIT protocol did not affect negatively this group, once these are both histological features of dystrophic muscle at the studied age. The mean values of the minimal Feret's diameter was also used recently by Nghiem et al. (2017) to attest the hypertrophy of tibial anterior dystrophic muscle of animals when studying the relationship between osteopontin, a multifunctional cytokine and myostatin, a known regulator of muscle mass.

In severe muscular dystrophies, muscle repair is uncontrolled and the primary defect leads to continuous cycles of myofiber degeneration and regeneration such that the muscle repair process is unable to replace the damaged muscle with new fibers. Fibrosis results when skeletal muscle is damaged and the regenerative process fails to recapitulate normal development (Lieber and Ward, 2013). At 16 weeks old, it was observed that regeneration process was highly present than fibrosis in both groups and the LIT protocol here employed has decreased the progression of muscular fibrosis related to dystrophy pathology on exercised mdx mice.

Fibroblasts contribute to the formation of a permanent fibrotic tissue by producing an accumulation of fibrotic interstitial ECM components such as hyaluronic acid, fibronectin, proteoglycans and interstitial collagens (Gillies et al., 2017; Serrano and MuñozCánoves, 2010), such as collagen types I and III (Mann et al., 2011). Fibrosis can be considered an aberrant repair or dysregulated tissue repair response that has severe consequences in muscular dystrophies. Also, collagen content is known to diminish myoblast growth and differentiation and the presence of collagen I at interstitial ECM of injured muscle can affect muscle repair (Mann et al., 2011).

Muscle fibrosis deposition seems to have either a morphological and functional consequence for muscular dystrophies. As described by Mackey et al. (2005), types I and III collagen coexist in the same tissue and it seems that their relative proportions affect the overall functional characteristics of that tissue. Animals that underwent the LIT protocol have demonstrated positive adaptations regarding the presence in the endomysial structure of these two types of collagen: type III collagen was higher reacted and type I poor reacted in TA muscle of exercised group when compared to dystrophic animals of control group. This organization related to exercise of these two different types of collagen can be named as positive because type III collagen is present initially in wound healing and regenerating skeletal muscle and type I collagen is present in latter stages of regeneration (Lehto et al., 1985) and during aging (Wood et al., 2014). It reinforce that exercise extended the dystrophic muscle regeneration process and delayed fibrosis deposition as was also demonstrated by data of CLN and amount of collagen fibers on muscular fibrosis.

Instead fibrosis is demonstrated by large increase in collagens types I and III in muscle ECM (Mann et al., 2011), Smith and Barton (2014) conclude that muscle fibrosis does not lead to increased passive stiffness and that collagen content is not predictive of muscle stiffness. It was demonstrated that increased tissue stiffness in skeletal muscle fibrosis is not simply explained by increased collagen crosslinks, suggesting collagen organization may affect tissue stiffness (Chapman et al., 2015; Lieber and Ward, 2013). Gillies et al. (2017) using scanning electron microscopy described recently the structure and composition of wild-type and fibrotic ECM. They have shown that collagen in the ECM is organized into large bundles of fibrils, or collagen cables, and the number of these cables (but not their size) increases in desmin knockout muscle (a fibrosis model). The increase in cable number is accompanied by increased muscle stiffness and an increase in the number of collagen producing cells.

Immunohistochemistry is a morphology-based technique (Leong et al., 2010) that can help to gain insight into collagen types location in established dystrophy animals submitted to LIT exercise protocol. The content of collagen types is important to infer which type is the most present in the studied muscle. Although, we must also know where and how collagen types are organized and how they work and adapt together in dystrophic muscle (Aurora et al., 2014).

The mainly limitation of this study is that one single muscle was here described. TA muscle was selected by the reasons already listed in discussion section and also to enable translational results with other research groups and previous published articles on $\mathrm{mdx}$ 
mouse.

Our results have shown that horizontal treadmill LIT protocol leads to positive repair of TA muscle delaying fibrosis deposition and extending dystrophic muscle regeneration in $\mathrm{mdx}$ mice. To find an ideal threshold of exercise to mdx mice muscle morphology and function is extremely important once the intensity can be further translated to human boys with DMD and also to be employed by physiotherapist who will soon work with new genetic therapies which have the aim of restore dystrophic muscle.

\section{CONFLICT OF INTEREST}

No potential conflict of interest relevant to this article was reported.

\section{ACKNOWLEDGMENTS}

This research was supported by FAPEMIG (Minas Gerais State Agency for Research and Development) process n ${ }^{\circ}$ APQ-0197112. The funders had no role in study design, data collection and analysis, decision to publish or preparation of the manuscript.

\section{REFERENCES}

Ardite E, Perdiguero E, Vidal B, Gutarra S, Serrano AL, Muñoz-Cánoves P. PAI-1-regulated miR-21 defines a novel age-associated fibrogenic pathway in muscular dystrophy. J Cell Biol 2012;196:163-175.

Augusto V, Padovani CR, Campos GE. Skeletal muscle fiber types in c57bl6j mice. Braz J Morphol Sci 2004;21:89-94.

Aurora A, Garg K, Corona BT, Walters TJ. Physical rehabilitation improves muscle function following volumetric muscle loss injury. BMC Sports Sci Med Rehabil 2014;6:41.

Baltgalvis KA, Call JA, Cochrane GD, Laker RC, Yan Z, Lowe DA. Exercise training improves plantar flexor muscle function in $\mathrm{mdx}$ mice. Med Sci Sports Exerc 2012;44:1671-1679.

Briguet A, Courdier-Fruh I, Foster M, Meier T, Magyar JP. Histological parameters for the quantitative assessment of muscular dystrophy in the mdx-mouse. Neuromuscul Disord 2004;14:675-682.

Bushby K, Finkel R, Birnkrant DJ, Case LE, Clemens PR, Cripe L, Kaul A, Kinnett K, McDonald C, Pandya S, Poysky J, Shapiro F, Tomezsko J, Constantin C; DMD Care Considerations Working Group. Diagnosis and management of Duchenne muscular dystrophy, part 2: implementation of multidisciplinary care. Lancet Neurol 2010;9:177-189.

Bushby K, Finkel R, Wong B, Barohn R, Campbell C, Comi GP, Connolly AM, Day JW, Flanigan KM, Goemans N, Jones KJ, Mercuri E, Quin- livan R, Renfroe JB, Russman B, Ryan MM, Tulinius M, Voit T, Moore SA, Lee Sweeney H, Abresch RT, Coleman KL, Eagle M, Florence J, Gappmaier E, Glanzman AM, Henricson E, Barth J, Elfring GL, Reha A, Spiegel RJ, O'donnell MW, Peltz SW, Mcdonald CM; PTC124-GD007-DMD STUDY GROUP. Ataluren treatment of patients with nonsense mutation dystrophinopathy. Muscle Nerve 2014;50:477-487.

Call JA, McKeehen JN, Novotny SA, Lowe DA. Progressive resistance voluntary wheel running in the mdx mouse. Muscle Nerve 2010;42: 871-880.

Capogrosso RF, Mantuano P, Cozzoli A, Sanarica F, Massari AM, Conte E, Fonzino A, Giustino A, Rolland JF, Quaranta A, De Bellis M, Camerino GM, Grange RW, De Luca A. Contractile efficiency of dystrophic $\mathrm{mdx}$ mouse muscle: in vivo and ex vivo assessment of adaptation to exercise of functional end points. J Appl Physiol (1985) 2017;122:828843.

Carter GT, Abresch RT, Fowler WM Jr. Adaptations to exercise training and contraction-induced muscle injury in animal models of muscular dystrophy. Am J Phys Med Rehabil 2002;81(11 Suppl):S151-161.

Chapman MA, Pichika R, Lieber RL. Collagen crosslinking does not dictate stiffness in a transgenic mouse model of skeletal muscle fibrosis. J Biomech 2015;48:375-378.

Cup EH, Pieterse AJ, Ten Broek-Pastoor JM, Munneke M, van Engelen BG, Hendricks HT, van der Wilt GJ, Oostendorp RA. Exercise therapy and other types of physical therapy for patients with neuromuscular diseases: a systematic review. Arch Phys Med Rehabil 2007;88:14521464 .

De Brito MK, Camargo Filho JC, Vanderlei LC, Tarumoto MH, Dal Pai V, Giacometti JA. Dimensões geométricas das fibras do músculo sóleo de ratos exercitados em esteira rolante: a importância da análise por meio de imagens digitalizadas. Rev Bras Med Esporte 2006;12:103-107.

Dellorusso C, Crawford RW, Chamberlain JS, Brooks SV. Tibialis anterior muscles in mdx mice are highly susceptible to contraction-induced injury. J Muscle Res Cell Motil 2001;22:467-475.

Dubach-Powell J. DMD_M.1.2.001: Quantitative determination of muscle fiber diameter (minimal Feret's diameter) and percentage of centralized nuclei [Internet]. Newcastle (UK): TREAT-NKD; 2014 [cited 2015 Mar 15]. Avaiable from: http://www.treat-nmd.eu/downloads/file/ sops/dmd/MDX/DMD_M.1.2.001.pdf.

Ferry A, Benchaouir R, Joanne P, Peat RA, Mougenot N, Agbulut O, Butler-Browne G. Effect of voluntary physical activity initiated at age 7 months on skeletal hindlimb and cardiac muscle function in mdx mice of both genders. Muscle Nerve 2015;52:788-794.

Frinchi M, Macaluso F, Licciardi A, Perciavalle V, Coco M, Belluardo N, Morici G, Mudò G. Recovery of damaged skeletal muscle in $\mathrm{mdx}$ mice through low-intensity endurance exercise. Int J Sports Med 2014;35:19- 
27.

Gaiad TP, Araujo KP, Caromano FA, Ambrosio CE. Duchenne muscular dystrophy: experimental models on physical therapy. In: Hegde M, Ankala A, editors. Muscular dystrophy. Rijeka (Croatia): InTech; 2012. p. 535-544.

Gaiad TP, Araujo KP, Serrão JC, Miglino MA, Ambrósio CE. Motor physical therapy affects muscle collagen type I and decreases gait speed in dystrophin-deficient dogs. PLoS One 2014;9:e93500.

Gianola S, Pecoraro V, Lambiase S, Gatti R, Banfi G, Moja L. Efficacy of muscle exercise in patients with muscular dystrophy: a systematic review showing a missed opportunity to improve outcomes. PLoS One 2013;8:e65414.

Gillies AR, Chapman MA, Bushong EA, Deerinck TJ, Ellisman MH, Lieber RL. High resolution three-dimensional reconstruction of fibrotic skeletal muscle extracellular matrix. J Physiol 2017;595:1159-1171.

Gosselin LE, Williams JE, Personius K, Farkas GA. A comparison of factors associated with collagen metabolism in different skeletal muscles from dystrophic (mdx) mice: impact of pirfenidone. Muscle Nerve 2007;35:208-216

Grange RW. DMD_M.2.1.001: Use of treadmill and wheel exercise for impact on mdx mice phenotype [Internet]. Newcastle (UK): TREAT-NKD; 2014 [cited 2017 Mar 15]. Available from: http://www.treat-nmd.eu/ downloads/file/sops/dmd/MDX/DMD_M.2.1.003.pdf.

Grounds MD, Radley HG, Lynch GS, Nagaraju K, De Luca A. Towards developing standard operating procedures for pre-clinical testing in the mdx mouse model of Duchenne muscular dystrophy. Neurobiol Dis 2008;31:1-19.

Haas M, Vlcek V, Balabanov P, Salmonson T, Bakchine S, Markey G, Weise M, Schlosser-Weber G, Brohmann H, Yerro CP, Mendizabal MR, Stoyanova-Beninska V, Hillege HL. European Medicines Agency review of ataluren for the treatment of ambulant patients aged 5 years and older with Duchenne muscular dystrophy resulting from a nonsense mutation in the dystrophin gene. Neuromuscul Disord 2015;25:5-13.

Huebner KD, Jassal DS, Halevy O, Pines M, Anderson JE. Functional resolution of fibrosis in $\mathrm{mdx}$ mouse dystrophic heart and skeletal muscle by halofuginone. Am J Physiol Heart Circ Physiol 2008;294:H15501561.

Hyzewicz J, Tanihata J, Kuraoka M, Ito N, Miyagoe-Suzuki Y, Takeda S. Low intensity training of mdx mice reduces carbonylation and increases expression levels of proteins involved in energy metabolism and muscle contraction. Free Radic Biol Med 2015;82:122-136.

Hyzewicz J, Tanihata J, Kuraoka M, Nitahara-Kasahara Y, Beylier T, Ruegg UT, Vater A, Takeda S. Low-intensity training and the C5a complement antagonist NOX-D21 rescue the mdx phenotype through modulation of inflammation. Am J Pathol 2017;187:1147-1161.
Kaczor JJ, Hall JE, Payne E, Tarnopolsky MA. Low intensity training decreases markers of oxidative stress in skeletal muscle of $\mathrm{mdx}$ mice. Free Radic Biol Med 2007;43:145-154.

Kjaer M, Magnusson P, Krogsgaard M, Boysen Møller J, Olesen J, Heinemeier K, Hansen M, Haraldsson B, Koskinen S, Esmarck B, Langberg $\mathrm{H}$. Extracellular matrix adaptation of tendon and skeletal muscle to exercise. J Anat 2006;208:445-450.

Lehto M, Duance VC, Restall D. Collagen and fibronectin in a healing skeletal muscle injury. An immunohistological study of the effects of physical activity on the repair of injured gastrocnemius muscle in the rat. J Bone Joint Surg Br 1985;67:820-828.

Leong TY, Cooper K, Leong AS. Immunohistology: past, present, and future. Adv Anat Pathol 2010;17:404-418.

Lieber RL, Ward SR. Cellular mechanisms of tissue fibrosis. 4. Structural and functional consequences of skeletal muscle fibrosis. Am J Physiol Cell Physiol 2013;305:C241-252.

Lynch GS, Hinkle RT, Chamberlain JS, Brooks SV, Faulkner JA. Force and power output of fast and slow skeletal muscles from mdx mice 6-28 months old. J Physiol 2001;535(Pt 2):591-600.

Mackey AL, Donnelly AE, Roper HP. Muscle connective tissue content of endurance-trained and inactive individuals. Scand J Med Sci Sports 2005;15:402-408.

Mann CJ, Perdiguero E, Kharraz Y, Aguilar S, Pessina P, Serrano AL, MuñozCánoves P. Aberrant repair and fibrosis development in skeletal muscle. Skelet Muscle 2011;1:21.

Manning J, O'Malley D. What has the mdx mouse model of Duchenne muscular dystrophy contributed to our understanding of this disease? J Muscle Res Cell Motil 2015;36:155-167.

Markert CD, Ambrosio F, Call JA, Grange RW. Exercise and Duchenne muscular dystrophy: toward evidence-based exercise prescription. Muscle Nerve 2011;43:464-478.

Nghiem PP, Kornegay JN, Uaesoontrachoon K, Bello L, Yin Y, Kesari A, Mittal P, Schatzberg SJ, Many GM, Lee NH, Hoffman EP. Osteopontin is linked with AKT, FoxO1, and myostatin in skeletal muscle cells. Muscle Nerve 2017;56:1119-1127.

Pertl C, Eblenkamp M, Pertl A, Pfeifer S, Wintermantel E, Lochmüller H, Walter MC, Krause S, Thirion C. A new web-based method for automated analysis of muscle histology. BMC Musculoskelet Disord 2013; 14:26.

Schill KE, Altenberger AR, Lowe J, Periasamy M, Villamena FA, RafaelFortney JA, Devor ST. Muscle damage, metabolism, and oxidative stress in mdx mice: Impact of aerobic running. Muscle Nerve 2016; 54:110-117.

Serrano AL, Muñoz-Cánoves P. Regulation and dysregulation of fibrosis in skeletal muscle. Exp Cell Res 2010;316:3050-3058. 
Smith LR, Barton ER. Collagen content does not alter the passive mechanical properties of fibrotic skeletal muscle in mdx mice. Am J Physiol Cell Physiol 2014;306:C889-898.

Smythe GM, White JD. Voluntary wheel running in dystrophin-deficient (mdx) mice: Relationships between exercise parameters and exacerbation of the dystrophic phenotype. Version 3. PLoS Curr 2011 Dec 18 [revised 2012 Mar 19];3:RRN1295.

Turgeman T, Hagai Y, Huebner K, Jassal DS, Anderson JE, Genin O, Nagler A, Halevy O, Pines M. Prevention of muscle fibrosis and improvement in muscle performance in the $\mathrm{mdx}$ mouse by halofuginone. Neuromuscul Disord 2008;18:857-868.
Vainzof M, Ayub-Guerrieri D, Onofre PC, Martins PC, Lopes VF, Zilberztajn D, Maia LS, Sell K, Yamamoto LU. Animal models for genetic neuromuscular diseases. J Mol Neurosci 2008;34:241-248.

Willmann R, De Luca A, Benatar M, Grounds M, Dubach J, Raymackers JM, Nagaraju K; TREAT-NMD Neuromuscular Network. Enhancing translation: guidelines for standard pre-clinical experiments in $\mathrm{mdx}$ mice. Neuromuscul Disord 2012;22:43-49.

Wood LK, Kayupov E, Gumucio JP, Mendias CL, Claflin DR, Brooks SV. Intrinsic stiffness of extracellular matrix increases with age in skeletal muscles of mice. J Appl Physiol (1985) 2014;117:363-369. 